\title{
La escuela rural de Utawilaya: Una Educación liberadora desde Puno - Perú 1902
}

\author{
Utawilaya rural school: \\ A liberating Education in Puno - Peru 1902 \\ A escola rural de Utawilaya: \\ Una educação de liberação de Puno - Perú 1902 \\ David Ruelas Vargas ${ }^{1}$ \\ Universidad Nacional del Altiplano Puno (Perú)
}

Recepción: 21/05/2016

Evaluación: 23/06/2016

Aceptación: 09/07/2016

Artículo de Revisión

DOI: http://dx.doi.org/10.19053/01227238.4919

\section{RESUMEN}

En este manuscrito se presentan datos del desarrollo de la Escuela Rural Indígena de Utawilaya en el distrito de Chucuito Platera de la provincia de Puno - Perú en 1902. Frente a la exclusión educativa, económica, social y política hacia la población rural, por el Estado. El maestro Manuel Zúñiga Camacho fundó la Escuela Rural Indígena con una postura pedagógica hacia una educación liberadora. Zúñiga Camacho consideraba a la educación como la única forma de salvación de la exclusión, explotación, y pobreza, instituido por los oligarcas y gamonales. La historiografía peruana coincide en afirmar que uno de los movimientos más fuertes en la sierra sur a inicios del siglo XX fue la lucha por la educación, que se consideraba de privilegio para la clase alta o élite gobernante (oligárquico y gamonal). El presente estudio es realizado con base en documentos históricos inéditos, que contribuyen al estudio de la historia de Puno. Se ha aplicado la metodología y técnica de investigación de biblioteca, en la cual se revisan las fuentes primarias y secundarias que conducen al análisis

$1 \quad$ Magíster en Didáctica de las Ciencias Sociales, Docente de la Facultad de Ciencias de la Educación, Especialidad de Ciencias Sociales de la Universidad Nacional del Altiplano Puno - Perú. Email: divadrv@hotmail.com 
y crítica. La marginación de los pueblos indígenas no es un fenómeno actual, en el marco de la historia republicana se viene arrastrando desde hace doscientos años. En esta exclusión subyace el concepto que se manejó (y aún se maneja) sobre lo indígena, que se entiende como lo atrasado, lo ignorante, y por tanto no es incluido en las políticas de desarrollo del Estado peruano. Para (Aliaga 2015), la incidencia de la pobreza de acuerdo con o "según" la lengua materna de las personas, afecta más a la población que tiene como lengua aprendida en su niñez una lengua nativa: quechua o aimara. Así, en el año 2013, la pobreza afectó al 35,9\% de la población. La escuela Utawilaya su programa de alfabetización y lectoescritura abarcó a grupos de adultos y niños. La educación liberadora Manuel Zúñiga Camacho destaca aspectos trascendentales, tales como: liberar a la persona, transformar la comunidad, toma de conciencia, transformar la realidad en un ambiente sin discriminación, explotación, humillación y con derecho a educación.

Palabras clave: Discriminación, educación, enseñanza, escuela, exclusión, indígena, rural.

\section{ABSTRACT}

This paper details the development of the Indigenous and rural School Utawilaya in the district Chucuito Platera, in the province of Puno- Peru in 1902. Because of the educational, economic, social and political exclusion of the rural population by the State, the teacher Manuel Zuniga Camacho founded the Indigenous and rural School with a pedagogical stance towards a liberating education. Zúñiga
Camacho considered education as the only way of salvation from exclusion, exploitation and poverty; established by the oligarchs and political bosses. Peruvian historiography agrees that one of the strongest movements in the southern highlands in the early twentieth century was the struggle for education, which was considered as a privilege for the upper class or ruling elite (oligarchy and tyranny). This research work is based on unpublished historical documents, which contribute to the study of the history of Puno. Library research methods and techniques have been applied for reviewing primary and secondary sources leading to analysis and criticism. The marginalization of indigenous peoples is not a current phenomenon; it comes from two hundred years ago within the framework of the republican history. This exclusion underlies the perception (still current) of indigenous people as backward and ignorant, who therefore are not included in the development policies of the Peruvian State. For (Aliaga, 2015) the incidence of poverty, "on grounds of" mother tongue, affects mainly the population with a native language: Quechua or Aymara. Thus, in 2013, poverty affected $35.9 \%$ of the population. The Utawilaya school literacy program included groups of adults and children. The liberating education of Manuel Zúñiga Camacho highlights the following transcendental aspects: people's liberation, community transformation, awareness, change of reality into an environment without discrimination, exploitation, humiliation and entitled to education. 
Keywords: Discrimination, education, teaching, school, exclusion, indigenous, rural.

\section{RESUMO}

Neste manuscrito detalha o desenvolvimento da Escola Rural Indígena de Utawilaya no distrito Chucuito Platera, da província de Puno - Perú en 1902. De frente ao exclusão educacional, económico, social e política para a população rural, pela Estado. O professor Manuel Zuniga Camacho fundou a Escola Rural Indígena com uma postura pedagógica no sentido de uma educação libertadora. Zúñiga Camacho considerado a educação como o único caminho da salvação exclusão, exploração e pobreza, estabelecida pelos oligarcas e gamonales. A historiografia de Perú, concorda dizer que um dos movimentos mais fortes nas terras altas do Sul no início do século XX foi a luta pela educação, que foi considerado de privilegio para a classe alta ou elite dominante (oligárquica e gamonal). Este estudo é realizado com base em documentos históricos inéditos, que contribuem para o estudo da história de Puno. Foi usada a metodologia e técnica de pesquisa de biblioteca em que as fontes primárias e secundárias que levam a análise e crítica são revisados. A marginalização dos povos indígenas não é um fenômeno atual no âmbito da história republicana se arrasta por duzentos anos. Esta exclusão está na base do conceito foi tratado (e ainda funciona) sobre os indígenas, entendido como atrasado, ignorantes, e portanto não está incluído nas políticas de desenvolvimento do Estado peruano. Para (Aliaga 2015), a incidência da pobreza de acordo com ou "según" a língua materna das pessoas, afeta a população cuja língua aprendida na infância uma língua nativa quíchua ou aimará. Assim, em 2013, a pobreza foi afetado $35,9 \%$ da população. A Escola Utawilaya sua programa de alfabetização e lectoescritura abarcou grupos de adultos e crianças. A educação libertadora Manuel Zuniga Camacho destaques aspectos transcendentais tais como: liberar a pessoa, transformando a comunidade, consciência, transformar a realidade em um ambiente sem discriminação, exploração, humilhação e com direito a educação.

Palavras-chave: Discriminação, educação, ensino, escola, exclusão, indígena, rural.

\section{INTRODUCCIÓN}

Una de las características de la educación peruana en el ámbito rural andino, es su enorme exclusión. Puno es una de las provincias, con población Quechua y Aimara que fueron y son aisladas por las políticas de desarrollo del país, existen brechas de grandes disparidades educativas, sociales y económicas, en las que los pueblos indígenas andinos corresponden a los grupos más desfavorecidos y con los más bajos niveles de bienestar.

Puno a inicios del siglo XX fue uno de los departamentos de menor desarrollo industrial del Perú, tenía una economía agropecuaria poco tecnificada y una 
población mayoritariamente indígena y analfabeta; donde tres estamentos convivían bajo una violenta tensión social: el comunitario-indígena, el feudalismo-terrateniente y el capitalismo mercantilista comerciante, a ellos se sumaban el gamonalismo patriarcal y el clero católico de mayor predominio en el Perú y marcadamente conservador ${ }^{2}$. La educación nacional, por consiguiente, no tiene un espíritu nacional: tiene mas bien un espíritu colonial y colonizador. Cuando en sus programas de instrucción pública el Estado se refiere a los indios, no se refiere a ellos como a peruanos iguales a todos los demás. Los considera como una raza inferior ${ }^{3}$. Las minorías étnicas, o pueblos indígenas, más allá de su número o representatividad, ya no pueden ser reducidas a las "catacumbas", al silencio, a la exclusión y a la pobreza extrema, por el solo hecho de ser diferentes y no responder a las características específicas del proyecto nacional mestizocriollo construido a partir de un neocolonialismo, aún no sobrepasado 4 .

Lainvestigación referida es deimportancia porque se enfocó en la investigación de la historia de la educación rural en el Altiplano puneño, identificando el origen, el aporte y la influencia de la Escuela Rural de Utawilaya. La investigación realizada brinda la oportunidad de describir, interpretar y analizar el desarrollo de la educación rural, para poder generar teorías pedagógicas pertinentes a los cambios requeridos.

A lo largo del presente documento se demostrará el desarrollo de una educación liberadora rural indígena promovido por Manuel Zúñiga Camacho. El objetivo principal de la investigación es identificar el desarrollo de la Escuela Rural de Utawilaya y la educación liberadora en Puno de 1902, describir la exclusión económica, política, social, educativa e interpretar la influencia de la Escuela Rural en el Altiplano puneño en el contexto sociopolítico. La metodología utilizada es el estudio de biblioteca e investigación histórica que busca reconstruir el pasado de la manera más objetiva y exacta posible.

Desde el punto de vista teórico, es relevante porque se evidencia la existencia de una educación liberadora de Manuel Zúñiga Camacho desarrollada en la escuela rural de Utawilaya en el Altiplano puneño en 1902. La escuela rural de Utawilaya vendría a ser una de las precursoras de la pedagogía liberadora que Paulo Freire planteó, y que le da significancia al ambiente de la historia de la educación peruana, considerando que el mismo puede influir de forma directa en la pedagogía educativa de nuestro país.

En cuanto a la conclusión de la investigación se ha identificado la exclusión de una educación para los indígenas, por parte del Estado oligárquico, frente a ello,

2 Gustavo Aliaga Rodríguez, El Rijcharismo en el Altiplano peruano como una experiencia pionera de salud intercultural en América. "Rijcharismo" movement in the Peruvian High Plateau as a Pioneer Intercultural Health Experience in the Americas (Retrieved from, 2015), 497-509.

3 José Carlos Mariátegui La Chira, 7 Ensayos de interpretación de la realidad peruana. Vol. I (Ayacucho, Perú: Fundación Biblioteca Ayacucho, 1979), 89.

4 Sonia Comboni \& José Juáres J., Diversidad cultural, educación y democracia: etapas en la construcción de la educación indígena en América Latina (2001), 235-274. 
el gran maestro indígena Manuel Zúñiga Camacho fundó la primera Escuela Rural Indígena en el Altiplano puneño en el año 1902, para liberar a la población indígena de la explotación, racismo, exclusión educativa y humillación. El interés científico de la presente investigación, se basa en la importancia del origen de una educación liberadora plateado por Manuel Zúñiga Camacho, precursor de la educación rural en América Latina y de la pedagogía liberadora.

Los objetivos planteados para esta investigación fueron: Conocer el origen de la Escuela Rural de Utawilaya y al maestro indígena Manuel Zúñiga Camacho en Puno de 1902. Objetivos específicos: Explicar la exclusión económica, política, social, educativa y la Escuela Rural de Utawilya en el Altiplano puneño hacia una educación liberadora, identificar los aportes de la Escuela Rural de Utawilaya, identificar a los sujetos educativos de la Escuela Rural de Utawilaya e interpretar la influencia de la Escuela Rural de Utawilaya en el Altiplano puneño en el contexto sociopolítico.

\section{Material y método. Ámbito o alcance de estudio}

La investigación fue realizada, correspondiente al Distrito de Platería. Departamento y Provincia de Puno, ubicado en el Altiplano a una altura de 3 826 msnm a tres kilómetros del Lago Titicaca, al sureste de la ciudad de Puno.

El presente estudio es realizado con base en documentos históricos inéditos, que contribuyen al estudio de la historia de Puno. Se ha aplicado la metodología y técnica de investigación de biblioteca, en la cual se revisan las fuentes primarias y secundarias que conducen al análisis y crítica de las fuentes para luego procesar los datos.

Metodología de la investigación: es el estudio de biblioteca, se utiliza cuando ya existen en textos publicados y disponibles de otras maneras los datos que se habrán de recolectar y analizar en nuestro estudio. Hacemos investigación de biblioteca cuando el área del problema y la pregunta de nuestra investigación son tales que dependen del acceso a materiales relevantes publicados, y utilizamos la información que contienen como una base de datos para todo como su base de datos principal, y no se refiere a la investigación conducida solo en bibliotecas. La "investigación de biblioteca" es una simplificación para identificar cualquier investigación que utilice textos previamente producidos o "evidencia documental" .

Investigación histórica: este tipo de investigación busca reconstruir el pasado de la manera más objetiva y exacta posible, para lo cual de manera sistemática recolecta, evalúa, verifica y sintetiza evidencias que permitan obtener conclusiones válidas, a menudo derivadas de hipótesis. Este tipo de investigación depende de datos observados por otros, más que por el investigador mismo. Estos datos

5 Michele Knobel and Colin Lankshear, Tres Enfoques para la Investigación Educativa (Editorial UNAM - México, 2002), 95. 
son de dos clases: secundarias, derivadas de la observación y registro directo de acontecimientos por su autor; quien informa sobre las observaciones realizadas primeramente por otros. Las fuentes primarias son evidencias de primera mano y deben usarse preferentemente. Las fuentes deben someterse a dos tipos de crítica: la primera que determina la autenticidad del documento; y la crítica externa, que examina los posibles motivos, prejuicios y limitaciones del autor del documento que posiblemente lo hayan determinado a exagerar, distorsionar u omitir información 6 .

Periodo de estudio: La realización del estudio transcurrió en los meses de enero, febrero, abril y mayo del 2016.

\section{Pueblos indígenas a inicios del siglo XX: exclusión económica, política, social y educativa}

Tres grupos étnico-culturales coexisten en el espacio geográfico puneño: indígenas, mestizos y blancos; a su vez, los indígenas se dividen en quechuas y aimaras ${ }^{7}$. Al analizar la incidencia de la pobreza de acuerdo con o "según" la lengua materna de las personas, se observa que esta afecta más a la población que tiene como lengua aprendida en su niñez una lengua nativa: quechua o aimara.

Al finalizar el siglo XIX el proceso de expansión de las haciendas en el sur del Perú, iniciado tímidamente unas décadas atrás, se intensificó. Para contar con mano de obra, estas haciendas hicieron uso de la antigua figura del yanaconaje (donde el indígena quedaba como un sirviente del hacendado, a cambio de que este le diera algunos frutos de la cosecha) y el enganche (donde el indígena era traído a las haciendas para trabajar bajo un contrato que le restaba libertades $)^{8}$. Al empezar el siglo XX, Perú estaba gobernado por un pequeño grupo de familias aristocráticas dueñas de los medios de producción (la industria agropecuaria, las finanzas y la minería) con una limitada capacidad para industrializar el país y con un fuerte menosprecio hacia todas aquellas razas que no eran blancas. De 1895 a 1913, Puno experimentó una reactivación de su economía pecuaria. El comercio de lanas de camélidos, cuyo principal destino era el mercado europeo, alcanzó su mayor índice de exportación. Ante la demanda creciente del comercio internacional de lanas, los hacendados y gamonales del Altiplano puneño despojaron a las comunidades de sus tierras a través de la fuerza o de procesos judiciales apañados ${ }^{9}$. Desventajas que los pueblos indígenas padecieron en pobreza y explotación en casi toda la región andina de Puno - Perú.

6 Tamayo y Tamayo Mario, Aprender a investigar (Cali, Colombia. ICFES. 1998), 42.

7 Gustavo Aliaga Rodríguez, El Rijcharismo en el Altiplano peruano como una experiencia pionera de salud intercultural en América. "Rijcharismo" movement in the Peruvian High Plateau as a Pioneer Intercultural Health Experience in the Americas (2015), 497-509.

8 Jorge Alberto Cahuana, Repositorio de tesis de la Pontificia Universidad Católica del Perú (5 de noviembre, 2013), 45.

9 Gustavo Aliaga Rodríguez, El Rijcharismo en el Altiplano peruano como una experiencia pionera de salud intercultural en América. 
La población aimara y quechua del sur peruano, a inicios del siglo $X X$ fue obligada la población a prestar servicios gratuitos a los gamonales, alcaldes, jueces, al Gobernador y al Cura. En 1901 los indios de Santa Rosa de Juli, provincia de Chucuito, vienen en delegación a Lima a exponer sus quejas contra el subprefecto Don Mariano Vicente Cuentas, quien obliga a los doce ayllus de la localidad a realizar trabajos gratuitos para construir edificios públicos y casas de los gamonales ${ }^{10}$.

El concepto de República Aristocrática fue profundizado por los historiadores Alberto Flores Galindo y Manuel Burga en un libro publicado en 1980. En ese libro se razonaba sobre la naturaleza de la clase dominante peruana argumentando que no habría sido dirigente; es decir, que los poderosos habrían desplegado su liderazgo social y económico en forma cerrada y excluyente. ${ }^{11}$ De acuerdo con los autores, la denominada República Aristocrática (1895-1919) se caracterizó por el monopolio del poder político ejercido por una oligarquía conformada por miembros del Partido Civil, grupos económicos poderosos y terratenientes del interior del país ${ }^{12}$. Los grupos subalternos constituidos por el campesinado (mayoritariamente de origen indígena), los sectores populares urbanos, y las incipientes clases media y obrera fueron excluidas de toda forma de participación en la vida política. De esta manera, el Estado oligárquico civilista estuvo regido por una élite de composición social homogénea, la cual excluyó de forma sistemática a las mayorías nacionales y promovió un crecimiento económico basado en la exportación de materias primas y la promoción irrestricta de los capitales extranjeros.

Según Flores (1977) citado por Kopsoli (1977), de 1876 a 1915, el número de haciendas en Puno había aumentado de 705 a $3219 .{ }^{13}$ Esta expansión no contribuyó a la mayor productividad de la tierra ni una mejor calidad de la fibra de lana exportada. El estímulo del mercado en vez de convertir al terrateniente en un capitalista, reforzó su mentalidad feudal y su poder monopólico impidiendo el acceso de las comunidades indígenas al mercado pecuario.

Ya en 1895 se muestran signos de una reactivación de la exportación de lanas. Esta demanda externa de lanas estimula a los hacendados o gamonales del sur a expandir sus haciendas y a "comprar" coercitivamente la lana de los comuneros; y no a introducir cambios tecnológicos significativos en el manejo de pastos y ganado. Una de las formas que utilizan los gamonales para expandir sus haciendas es el despojo directo de las tierras de las comunidades ${ }^{14}$. Es así,

"Rijcharism" movement in the Peruvian High Plateau as a Pioneer Intercultural Health Experience in the Americas (2015), 497-509.

10 Grimaldo Antonio Rengifo, “Campesinos en Puno 1895 - 1925” (Tesis para optar el Título de LIMA, 1990), 23.

11 Manuel Burga y Alberto Flores Galindo, Apogeo y crisis de la República Aristocrática. quinta edición (Lima, ediciones Rikchay, Perú, 1990).

12 Jorge Basadre Grohmann, Historia de la República del Perú 1822-1933, quinta ed., Vol. 10. (Lima, Perú, ediciones Historia, 1963$), 587$.

13 Flores Galindo, La oligarquía arequipeña y los movimientos campesinos (estudiado con profundidad, 1977).

14 Grimaldo Antonio Rengifo Balarezo, "Exportación de lanas y movimientos campesinos" (Tesis para optar el Título de Licenciado en Sociología, 1990), 45. 
que veían a la ciudad de Puno, tan solo como un espacio extractivo de materia prima, además las autoridades políticas no propiciaron el enganche entre el auge de las lanas, el proceso modernizador de las haciendas y el desarrollo político y social del departamento ${ }^{15}$. Ante la demanda creciente del comercio internacional de lanas, los hacendados y gamonales del Altiplano puneño despojaron a las comunidades de sus tierras a través de la fuerza o de procesos judiciales apañados ${ }^{16}$.

Uno de los Lizares $^{17}$, precisamente, fue quien presentó al parlamento un proyecto de ley para erradicar a los indios. Por la misma fecha, los ingleses tuvieron a nivel de ideas erradicar a los indios de las altiplanicies de Cuzco y Puno para desarrollar capitalistamente la ganadería ovina, pero desistieron ${ }^{18}$. El problema de la tierra es en sí mismo un hecho económico, y está ligado a la inequidad en el acceso a la propiedad. La concentración de la tierra en unos pocos, quienes valiéndose del poder político y económico despojaron al indio de sus tierras; esta es la causa de la explotación que ha padecido durante años el indígena puneño ${ }^{19}$.

La educación escolar indígena en el período colonial, hasta las primeras décadas del siglo $X X$, se puede caracterizar por una violencia etnocéntrica explícita, que intenta imponer la cultura hegemónica sobre las poblaciones indígenas ${ }^{20}$. Al finalizar el siglo XIX: la respuesta de los indios ante el gamonalismo ha sido diversa: constitución de escuelas, defensa legal ante los poderes públicos, levantamientos campesinos, bandolerismo, etc. Los intentos por constituir escuelas deben ser tomados o "asumidos" como una de las actitudes defensivas de los indios. Ellos querían aprender a leer, escribir y a realizar cálculos económicos. Querían proveerse de instrumentos para ejercer las libertades civiles, concurrir directamente al mercado y recurrir directamente ante los poderes del Estado (Rengifo, 1990). Frente a la exclusión debe considerarse desde una profunda base social, política e ideológica, delimitando a la escuela como el sitio desde donde es posible crear las condiciones para eliminar las vías de discriminación existentes en la sociedad ${ }^{21}$.

15 Héctor Elías Núñez Núñez, "Presencia protestante en el altiplano peruano. Puno, 1898-1915. El caso de los Adventistas del Séptimo Día: actores y conflictos," (Tesis para optar el título profesional de Licenciado en Antropología en la Universidad Nacional Mayor de San Marcos, Lima, UNMSM, 2008), 47.

16 Gustavo Aliaga Rodríguez, El Rijcharismo en el Altiplano peruano como una experiencia pionera de salud intercultural en América. "Rijcharismo" movement in the Peruvian High Plateau as a Pioneer Intercultural Health Experience in the Americas (2015), 497-509.

17 Hernán Alberto Jove Quimper, Poder Patrimonial y Dominio Simbólico Iglesia "Tintiri”, Azangaro-Puno: 1860-1968. Lizares Terrateniente de Azángaro: fanático religioso, maniático por el dinero y despojador de tierras. (Rev. Investig. Altoandin. 2016, vol 18 No. 1), $93-102$.

18 Grimaldo Antonio Rengifo, “Campesinos en Puno 1895 - 1925” (Tesis para optar el Título de LIMA, 1990).

19 Oscar Raúl Sandoval Zúñiga, El problema de la tierra y la cuestión indígena en la obra siete ensayos de interpretación de la realidad peruana de José Carlos Mariátegui (2004), 271-287.

20 Vera María Ferrão Candau, Educación intercultural en América Latina: Distintas concepciones y tensiones actuales. Estudios Pedagógicos, XXXVI, No 2, (2010), 333-342.

21 Isaac Irán Cabrera Ruiz y Teresita de Jesús Gallardo López, Educación intercultural del estudiante universitario: el enfoque de formación humanístico-intercultural. Revista Electrónica "Actualidades Investigativas en Educación" (2013), 34. 
Actualmente los pueblos indígenas de la región Puno siguen en abandono y en subdesarrollo. Así, en el año 2013, la pobreza afectó al 35,9\% (9,0\% pobre extremo y $26,9 \%$ pobre no extremo). Puno sigue siendo una de las regiones menos desarrolladas y pobres de Perú. El intervalo de pobreza monetaria en el 2013 fue de 29,5 - 32,4 \%. La mayor incidencia de pobreza se relacionó con la condición étnica (quechuas y aimaras) ${ }^{22}$.

En la actualidad se repiten las mismas políticas de exclusión social, educativa, y económica. Según la información propagada por el INEI $^{23}$, en el año 2009 la pobreza afectó al $34,8 \%$, de la población total, siendo las poblaciones de la sierra $(23,8 \%)$ y de la selva $(16,9 \%)$ los lugares de mayor incidencia. A nivel nacional las regiones más pobres se ubican en las regiones ubicadas en la sierra: Huancavelica, con una taza de pobreza del 77,2\%, seguido de Apurímac con un $70,3 \%$, Huánuco con $64,5 \%$, Ayacucho con $62,6 \%$ y Puno con un $60,8 \%$. Siendo Puno la quinta región más pobre del país.

\section{Origen de la escuela rural de Utawilaya hacia una educación liberadora}

Los campesinos eran convencidos para vender sus tierras a los hacendados, se fraguaban documentos falsos, pues se hacía aparecer que recibían sumas equitativas de dinero, cuando esto no era cierto. En consecuencia, los escritos eran medio engaño y estafa. Esto concitó a los campesinos la idea de que ellos y sus hijos deberían aprender a leer y escribir. Manuel Camacho captó esta inquietud en $1902^{24}$. Con el fin de obtener escuelas, los indios ${ }^{25}$ año tras año, han elevado solicitudes al Supremo Gobierno para que les designen solamente profesores; puesto que ellos aportaban el terreno, el local y el mobiliario de la escuela, pese a esos aportes, sus peticiones resultaron infructuosas por interposición de los gamonales. Y es más, cuando lograban persuadir a algún "misti" para que les oficiara de profesor, los gamonales trataban de disuadirlo por cualquier medio; y si aún se mantenía firme el profesor, entonces incendiaban y arrasaban la escuela ${ }^{26}$.

En el curso de asistir a la escuela primaria metodista en Iquique, servir en el ejército en Arequipa, viajar por México, California y Chile, los contactos de Camacho con el protestantismo y la educación, reforzaron su espíritu vehemente a independiente y alimentaron su pasión para mediar la salvación a los campesinos del Altiplano: "El único camino seguro de salvación de la

22 Instituto Nacional de Estadística e Informática, Informe Técnico (2014), 22.

23 Instituto Nacional de Estadística e Informática, Informe Técnico (2009), 45.

24 José Portugal Catacora, Historia de la Educación en Puno (Universidad Nacional del Altiplano, Puno, 2013), 52.

25 Karen Spalding, De Indios a Campesinos, (1974). El término "Indio", un término europeo, era aplicado en el tiempo de la conquista española a cualquiera que había vivido en las Américas antes de la llegada de los europeos.

26 Grimaldo Antonio Rengifo Balarezo, “Exportación de lanas y movimientos campesinos” (Tesis para optar el Título de Licenciado en Sociología, 1990), 47. 
postración en que se encuentra, es aprender a leer ${ }^{27}$. La escuela liberadora de Utawilaya, se fundó en el año 1902, fue la primera escuela donde se enseñó a los indígenas aimaras a leer y escribir, es decir alfabetizar, entonces la posición de Manuel Zúñiga Camacho fue "la educación como práctica de la libertad".

Utahui-laya es una pequeña comunidad situada en las proximidades del distrito de Platería (en Chucuito), la primera escuela indígena en Puno y una de las primeras en América del Sur. La conforman pocas viviendas y consiguientemente pocas familias que huyeron de la voracidad de los hacendados, es este el caso de Agustín Tovar, dueño de las haciendas de Collacachi y Viluyo, entre las cuales se encontraba la primitiva vivienda de los familiares del fundador de la escuela, Manuel Camacho, el precursor del indigenismo, el Espartaco de su raza. La escuela fue establecida allí con esperanzados objetivos de enseñar a leer y escribir, instalar el adventismo, liberar a los suyos y elevar su cultura ${ }^{28}$.

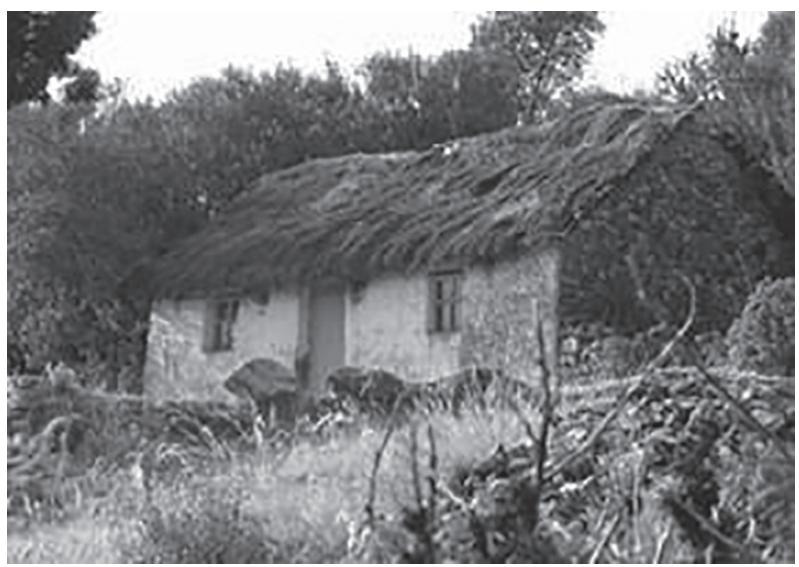

Imagen 1. Escuela de Utawilaya de Manuel Zúñiga Camacho construido en 1902, de material de adobe e $\mathrm{Ichu}^{29}$.

Fuente: Escuela de Utawilaya de Manuel Zúñiga Camacho construido en 1902, disponible en ${ }^{30}$.

El gobernador del Pueblo de Chucuito obligaba a los indígenas ayllus y comunidades a prestar servicios gratuitos, como Jilaqatas a los hombres casados, que deberían servir como auxiliar de las autoridades, por el periodo de un año. Los jueces de paz también tenían sus sirvientes llamados alguaciles, que

27 Allpanchis, Poder y Autoridad En Los Andes (Sicuani - Cusco: Instituto De Pastoral Andina, 1989), 67.

28 José Portugal Catacora, Blog Portugal (30 de Octubre de 2012) http://joseportugalcatacora.blogspot.pe/2012/10/manuel-z-camacho. html (01 de Mayo, 2016).

29 También conocido como Hiru ichu, paja brava. alimento natural de llamas, alpacas y vicuñas. Útil en la elaboración de escobas, sogas y en la elaboración de adobes de construcción. Fichas botánicas de especies agroforestales nativas aptas para tierras altoandinas. Ficha No. 11. (Care Bolivia, 2011).

30 Municipalidad de Plateria, Reseña histórica de la primera escuela rural Utawilaya - 1902. (Puno: 04 Abril 2014) http://www.plateria. org/index.php/171-resena-historica-de-la-primera-escuela-rural-utawilaya-1902. 
cumplían sus mandatos. El Tata-cura de la parroquia tenía sus mayordomos al servicio del Tata-cura del pueblo (o de los pueblos). Fuera de estos cargos, para las fiestas patronales, los campesinos obligatoriamente pasaban como alferados. Para la misa tenían que dar al Tata-cura un carnero degollado, gallinas, conejos, papas, quinua y huevo.

La entrevista a Manuel Zúñiga Camacho citada por Gallegos (1993) señala: "en el año 1902 fundé la primera escuela rural en el departamento de Puno. La escuela funcionaba en mi casa (en la casa de mi padrastro) en la parcialidad de Utawilaya. El local era una amplia sala que construí con ese propósito. Enseñaba a mis discípulos (hombres y mujeres y niños de toda edad) la lectura y la escritura del idioma castellano y la interpretación y el estudio de la Biblia" ${ }^{\prime 1}$.

Tamayo (1982) señala: “La escuela rural Utawilaya de Platería, fundada originalmente por Manuel Zúñiga Camacho Alca y que después se difundió por el Altiplano gracias a los esfuerzos de Fernando A. Stahl y Pedro Kalbermater, la educación indígena se inició en Puno con resultados insospechados y trascendentales". "Por primera vez el indio accedió de su propia dignidad. En 1902, el cacique adventista Manuel Zúñiga Camacho fundó la primera escuela rural en la parcialidad de Utawi laya (Platería), donde enseñó a leer y escribir, a interpretar la Biblia, la higiene personal y además atendía a los enfermos" ${ }^{\prime 32}$.

En 1902, a consecuencia de la solicitud de los comuneros de Utawilaya, el maestro Manuel Z. Camacho fundó la "Escuela particular de Indígenas de la parcialidad de Platería", en una época en que la escuela occidental se encontraba todavía lejos de penetrar en las áreas indígenas del altiplano. La finalidad de la escuela era la enseñanza de la lectoescritura en castellano y la capacitación en técnicas de cultivos y de salud. Si bien el objetivo de la escuela era la enseñanza del castellano, al parecer, para lograr un mejor rendimiento en la escuela, se hacía uso también del aymara en forma instrumental. Esto bastó para que la escuela fuera clausurada a causa de la reacción de los gamonales locales que la acusaron de incitar a la rebelión indígena. Sin embargo, Camacho, a través del apoyo de la Asociación Proindígena y de la Misión Adventista, logró reabrir su escuela en Utawilaya y posteriormente establecer en Platería una escuela más grande y de mayor cobertura ${ }^{33}$.

En la labor educativa liberadora emprendida por Manuel Z. Camacho destaca la liberación de la dominación y el esclavismo imperante, implantado por los gamonales bajo el sistema feudal. Camacho enseñó a leer y escribir, interpretar la historia; cambiar y transformar la estructura mental. Todos tienen derecho a la educación, sin excepción alguna de credo, religión, sexo, raza y otros. La

31 Luis Gallegos Arriola, Biografía de un Aymara (Puno, Universitaria, 1993), 46.

32 Gustavo Aliaga Rodríguez, El Rijcharismo en el Altiplano peruano como una experiencia pionera de salud intercultural en América. "Rijcharismo" movement in the Peruvian High Plateau as a Pioneer Intercultural Health Experience in the Americas (2015), 497-509.

33 Citarela Luca Amadio Massimo \& Zúñiga Madeleine, La educación indigena en América Latina, vol III. (Quito, Ecuador, ABYA AYALA - UNESCO 1990), 9. 
educación liberadora está comprometida en formar hombres y mujeres que sean fuertes para pensar y obrar; hombres y mujeres que sean poseedores y no esclavos de las circunstancias; hombres y mujeres que posean amplitud de mente; hombres y mujeres que posean claridad de pensamiento y valor para defender sus convicciones. Esta es la verdadera esencia de la pedagogía liberadora que propugnó Zúñiga Camacho, que tanta falta hace en nuestra sociedad actual. Camacho consideraba que la educación era la única forma de salvación del alma y un medio para liberarse de la opresión gamonal, del terrateniente y el sacerdote. En 1904, fue encarcelado por el poder político y obligado a abandonar la escuela.

Málaga (2014), afirma: Pero la oposición a la educación indígena era muy fuerte. Hoy se hace evidente que las razones para detener las iniciativas de cambio estaban motivadas por una élite que temía perder la base de su poder (la fuerza de trabajo gratuita de los indios). Esta escuela y Camacho sufrieron múltiples persecuciones por parte de los mestizos puneños y Camacho fue denunciado por provocar disturbios. Gallegos (1993) en el año de 1903 tuvo lugar un levantamiento de indios en el pueblo de Pomata, la sublevación dio lugar a un juicio militar para castigar a los rebeldes indios, Manuel Z. Camacho fue incluido en el juicio y recluido por dos años en la carceleta de la prefectura. Fue defendido por Teodomiro Gutiérrez Cuevas, Don Manuel Gonzales Prada. Desde la cárcel de Puno Camacho escribe varias cartas a Santiago de Chile y Buenos Aires quienes las defendieron en su revista "Las Señales de los tiempos" donde decían que Camacho no tenía mayor delito que el haber traído tardíamente la reforma al altiplano andino. En 1904, fue encarcelado por el poder político y obligado a abandonar la escuela ${ }^{34}$ (Aliaga Rodríguez, 2015).

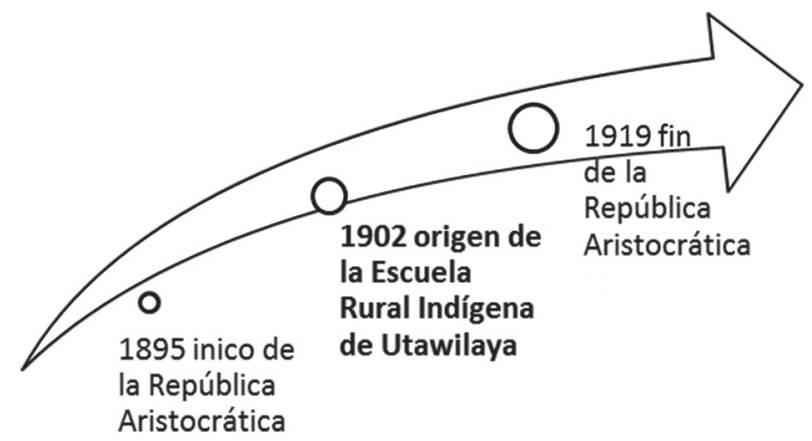

Figura 1. Origen de la Escuela Rural de Utawilaya en el proceso de desarrollo de la historia del Perú. (2016)

34 Gustavo Aliaga Rodríguez, El Rijcharismo en el Altiplano peruano como una experiencia pionera de salud intercultural en América. "Rijcharismo" movement in the Peruvian High Plateau as a Pioneer Intercultural Health Experience in the Americas (2015), 497-509. 
El Perú estaba gobernado entre 1895 y 1919 por un pequeño grupo oligárquico y gamonal, acuñado este periodo por Basadre como la República Aristocrática, dueña de los medios de producción (la industria agropecuaria, las finanzas y la minería), que despojaron de sus tierras al poblador rural, dando origen a inmensas haciendas, figura 1.

\section{Aporte y la enseñanza en la escuela rural de Utawilaya de Manuel Zúñiga Camacho}

Entrevista a Manuel Zúñiga Camacho citado por Gallegos (1993): Enseñaba a mis discípulos (hombres, mujeres y niños de toda edad) la lectura y la escritura del idioma castellano y la interpretación y el estudio de la Biblia. Además, curaba a los enfermos y daba orientaciones para la crianza de los animales y el cultivo de las plantas alimenticias. Según Portugal (2013) el maestro Manuel Zúñiga Camacho como hombre indígena que había alcanzado apreciables experiencia y cultura, pensó que el mejor camino para redimir a su pueblo era empezando a liberar las conciencias individuales de sus viejas costumbres por medio de la educación ${ }^{35}$.

Manuel Zúñiga sirvió en el ejército y viajó por los EE. UU., y Chile, consideraba que la educación era la única forma de salvación del alma y un medio para liberarse de la opresión del gamonal, el terrateniente y el sacerdote ${ }^{36}$. Camacho era un profesor múltiple, sabía curar a los enfermos, atendía consultas referentes a las enfermedades humanas, también absolvía consultas sobre los cultivos de las plantas alimenticias y hierbas medicinales. Daba consejos sobre la prevención de ciertas enfermedades. Además de enseñar a leer y escribir a sus alumnos, él estaba convencido del abuso de las autoridades de los pueblos, por el atraso y la ignorancia en que vivían los campesinos, además, porque no sabían expresarse en el idioma castellano.

La escuela que construyó Zúñiga Camacho, en la parcialidad de Utawilaya, fue con el propósito de enseñar la lectura, escritura del idioma castellano, interpretación, toma de conciencia y transformar la realidad, instruyó a sus hermanos indígenas de toda edad, con el propósito de liberarlos y transformar la realidad que vivía en un ambiente de explotación, humillación y discriminación, Figura 2.

35 José Portugal Catacora, Historia de la Educación en Puno (Puno, Perú, Corporación MERU E.I.R.L., 2013 ), 51.

36 Gustavo Aliaga Rodríguez, El Rijcharismo en el Altiplano peruano como una experiencia pionera de salud intercultural en América. "Rijcharismo" movement in the Peruvian High Plateau as a Pioneer Intercultural Health Experience in the Americas (2015), 497-509. 


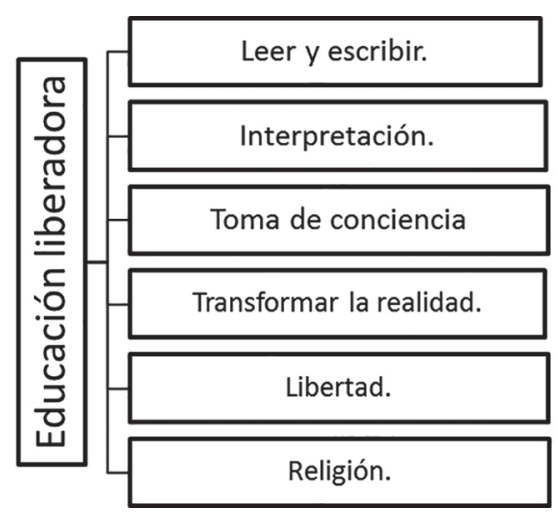

Figura 2. La enseñanza en la escuela rural de Utawilaya de Manuel Z. Camacho, (2016).

Entrevista a Manuel Zúñiga Camacho citado por Gallegos (1993) donde señala: en diciembre del año 1894, yo cumplía 24 años de edad para incorporarme al ejército revolucionario, decidí cambiar de apellido. El paterno Allqa lo reemplacé, por Zúñiga, en memoria del cura Zúñiga, mí recordado profesor de Historia Sagrada en la escuela Municipal de Moquegua; de mi madre tomé el segundo apellido: Camacho. Desde esta fecha en adelante yo seré, Manuel Zúñiga Camacho, o simplemente: Manuel Z. Camacho. El 17 de marzo de 1895, los montoneros intervinimos en la toma de Lima, por la entrada de Cocharcas, con 1,600 hombres y empezamos a combatir con los soldados de Cáceres $^{37}$. Manuel Z. Camacho se incorporó al ejército de Piérola para derrocar al presidente militar de Cáceres.

Para (Gallegos, 1993) en la entrevista realizada, el maestro indígena señaló lo siguiente: El retorno de Chile. El amor a la tierra, a mi comunidad decidí retornar, cuando tenía 27 años cumplidos. Llegué a Puno el 18 de enero de 1898, inmediatamente me puse a buscar a mis padres. Viajé a pie al lugar donde se encontraba mi comunidad, el ayllu Cutimbo. Fue grande mi sorpresa al encontrar solamente ruinas y vestigios de la casa destruida, con nuevo propietario del Doctor Agustín Tovar Arce.

En 1898 el regreso de Manuel Zúñiga Camacho a Puno fue dramático, su padre muerto, su comunidad absorbida como otras por la hacienda Collacachi, su madre estaba en segundas nupcias y ahora vivía dentro de la parcialidad de Utawilaya, cerca de Platería, adscrita a la provincia de Chucuito ${ }^{38}$.

Entrevista a Manuel Zúñiga Camacho citado por Gallegos (1993), el maestro indígena señaló: cuando estuve caminando por las calles de la ciudad de Puno, casualmente me encontré con Santiago Arpasi, quién me informó que mi

37 Luis Gallegos Arriola, Biografía de un Aymara (Puno, Universitaria, 1993), 43.

38 José Luis Rénique \& José Deustua. Intelectuales, indigenismo y descentralismo en el Perú, 1897-1931 (Cusco, Perú, Centro Bartolomé de las Casas 1984), 56. 
madre se encontraba en la parcialidad de Utawilaya, jurisdicción del distrito de Chucuito. Viajé a ese lugar. El encuentro con mi madre fue muy emotivo, de la alegría pasamos a las lágrimas. La abracé entre llorosos, ambos lloramos un largo momento, la encontré viejecita ella me dijo: tu padre ha muerto siendo colono en la hacienda de Collacachi, bajé en busca de libertad, ahora vivo en esta parcialidad, me he casado con Basilio Arpasi. Acepté quedarme con ellos, me puse a trabajar en chacra. En el año 1902 fundé la primera escuela rural en el departamento de Puno. La escuela funcionaba en la casa de mi padrastro en la parcialidad de Utawilaya.

\section{Manuel Zúñiga Camacho, el primer educador indígena}

Manuel Zúñiga Camacho, nace en 1871 en la comunidad indígena de Cutimbo en el distrito de Chucuito, hoy Cercado, en el departamento de Puno. Su madre era una indígena cusqueña de la entonces parcialidad de Anta, en el departamento de Cusco, traída a Puno a la edad de los 7 años para trabajar. Su padre Benedicto Luis Allca, indígena adscrito a esta parcialidad ${ }^{39}$.

En diciembre del año 1894, cumplió 24 años de edad y se incorporó al ejército revolucionario, decidió cambiar de apellido el de paterno Allqa lo reemplazó por Zúñiga, en memoria del cura Zúñiga, su recordado profesor de Historia Sagrada en la Escuela Municipal de Moquegua; de su madre tomó el segundo apellido: Camacho. Desde esta fecha en adelante se le conoce como Manuel Zúñiga Camacho, o simplemente: Manuel Z. Camacho. El 17 de marzo de 1895, los montoneros intervinimos en la toma de Lima, por la entrada de Cocharcas, con 1,600 hombres y empezamos a combatir con los soldados de Cáceres ${ }^{40}$.

En plena niñez deja Puno para ser encargado a su tío. Existen dos versiones que hablan de su destino en este período. La primera de ellas es que es entregado a su tío, el cual lo lleva a estudiar y trabajar a las salitreras de Iquique y que estudia en un colegio protestante, hasta su regreso a Puno ${ }^{41}$. La segunda versión, sus padres deciden enviarlo con sus tíos a la entonces ciudad de Moquegua, a estudiar para evitar que quedara como peón en la hacienda de Luis Tovar, hacendado puneño que había adjuntado las tierras de la comunidad indígena de Cutimbo como parte de sus tierras. Allí estudia y trabaja.

Otra de las versiones acerca de este hecho por Luis Gallegos sostiene que fueron sus padres quienes lo enviaron a estudiar a la ciudad de Moquegua, entregado por su padre a un arriero de camino a la ciudad de Moquegua.

39 Héctor Elías Núñez Núñez, "Presencia protestante en el altiplano peruano. Puno, 1898-1915. El caso de los Adventistas del Séptimo Día: actores y conflictos" (Tesis para optar el título profesional de Licenciado en Antropología en la Universidad Nacional Mayor de San Marcos, Lima, UNMSM., 2008), 153.

40 Luis Gallegos, Biografía de un Aymara (Puno, Universitaria, 1993), 27.

41 Juan Bautista Augusto Kessler, Historia de la Evangelización en el Perú (Lima, 1993), 223. 
Entrevista citada por Gallegos (1993) señala Manuel Z. Camacho "El amor a la tierra, a mi comunidad decidí retornar, tenía 27 años cumplidos. Llegué a Puno el 18 de enero de 1898. Inmediatamente me puse a buscar a mis padres. Viajé a pie al lugar donde se encontraba mi comunidad, el ayllu Cutimbo. Fue grande mi sorpresa al encontrar solamente ruinas y vestigios de la casa destruida, perteneciendo a la hacienda del Doctor Agustín Tovar Arce.

Camacho regresa a Puno en 1898. Como señala Renique (2004) el regreso de Camacho a Puno fue dramático, su padre muerto, su comunidad absorbida como otras por la hacienda Collacachi, su madre estaba en segundas nupcias y ahora vivía dentro de la parcialidad de Utawilaya, cerca de Platería, adscrita a la provincia de Chucuito.

Alrededor de 1902, Manuel Z. Camacho inicia su 'Escuela Libre' aunque clandestinamente, Camacho trabajaba ya con adultos desde el año 1898" Primero en la casa de su madre y luego al casarse con Paula Salas en la casa de su suegro ${ }^{42}$. Manuel Zúñiga Camacho tenía la idea clara sobre la importancia de la educación, había viajado a Estados Unidos, Chele y Lima. Se propuso de alfabetizar y elevar el nivel cultural de los indígenas que a través de la educación lograra, ensayó esa gran tarea en Utawilaya y luego empezó a propagarse por toda la región de Puno

En 1902 Manuel Zuñiga Camacho funda la primera escuela liberadora de la opresión y esclavismo. Primera escuela donde se enseñó al indígena aimara a leer y escribir. Después de 58 años de ardua lucha y tenas resistencia surge en 1961 revoluciona el mundo latinoamericano la teoría pedagógica de Paulo Freire sustentada en su obra "Pedagogía de la Liberación" pedagogía del oprimido (1970) "Educación como práctica de la libertad" y otras interesadas en la problemática educativa.

\section{La influencia de la Escuela Rural de Utawilaya en el contexto sociopolítico}

La penetración de los adventistas en Puno, data de 1913. Ese mismo año se suscita la querella del indio Manuel Zúñiga contra el obispo Valentín Ampuero a raíz de la instalación en la parcialidad de Platería del misionero adventista Fernando Stahl y de la creación de una escuela. A raíz de su prédica, los indios de dicha parcialidad no aceptaban realizar servicios sin paga a las autoridades del distrito. Esto genera la ira de los gamonales y autoridades ${ }^{43}$. Simpatizante del adventismo, en cuya doctrina funda su propio proyecto pedagógico o educativo.

La escuela de platería sin gran mobiliario, dio frutos de inestable valor. Los protestantes de Platería aprendieron a leer pronto y comprender por medio de

\footnotetext{
42 Wilbur Rubén Ayma Flores, Educación e Iglesia Adventista a Inicios del Siglo XX y Proyección al Tercer Milenio. En: Impacto Educativo. Hacia una Educación centrda en Valores. No. 1, Edición 1, (Puno, noviembre, 2000), 13.

43 Grimaldo Antonio Rengifo Balarezo, Campesinos en Puno 1895 - 1925 (Lima, 1990), 34.
} 
la lectura, la verdad del evangelio y la historia de la inquisición para apartarse del culto católico y profesar la pura doctrina de Cristo. Esos indios de Platería se civilizaron prontamente dando al vecindario clerical de Chucuito y Ácora ejemplos de civismo ${ }^{44}$. Cuando Camacho, a pesar de todas las intimidaciones, reabrió su escuela y, con ayuda de los adventistas, fundó una casa para escolares, una farmacia y un centro misionero, el obispo de Puno, Valentín Ampuero, organizó un ataque al pueblo, destruyó las nuevas instalaciones e hizo detener a Camacho. El incidente de 1913 desató una gran controversia y provocó que el Congreso levantara, dos años después, la prohibición de los cultos no católicos. Pero el conflicto generado por la escuela de Platería profundizó también la distancia entre la Iglesia católica y el movimiento indigenista ${ }^{45}$ (Mires, 1989). Zúñiga abrazó las causas revolucionarias, presentó un sin número de monumentos conmemorativos, y dirigió numerosas delegaciones en nombre del movimiento indígena ${ }^{46}$

\section{CONCLUSIÓN}

A inicios del siglo XX en el sur del Perú, principalmente en el departamento de Puno, creció la exportación de materia prima como la fibra de ovino y auquénido, para las fábricas textiles inglesas, frente a ellos los gamonales empezaron a exportar tal materia utilizando la mano de obra de la población indígena. En este contexto fue creciendo la exclusión económica, política y educativa a grandes escalas. Los indígenas de las comunidades más afectadas por el gamonalismo son las que tienen los mejores pastizales y, por consiguiente, eran despojados de sus tierras a través de compras engañosas solo por no saber leer y escribir. Por lo demás, todos los indígenas fueron impedidos a emerger: los servicios gratuitos a las autoridades políticas, al gamonal y el pago de tributos a esas mismas autoridades, entre las cuales se encontraba la Iglesia Católica.

Los aportes o el propósito de la pedagógica o educación de Camacho de 1902 fue: Liberar a la persona de la opresión, la explotación y la esclavitud implantado por los gamonales y oligarcas. La escuela Utawilaya no solo dio a la niñez, el principal agente de cambio, sino que por el contrario, su programa de alfabetización y lectoescritura abarcó a grupos de adultos, mostrando así su visión de la necesidad de conocimientos tanto académicos como prácticos que mejoraran su vida cotidiana. La educación liberadora Manuel Zúñiga Camacho destaca aspectos trascendentales, tales como: liberar a la persona, transformar la comunidad, toma de conciencia, transformar la realidad en un ambiente

44 Julio Cano Periodico, La unión. Lo de la Plateria (23 de Enero, 1914), 2.

45 Mires Fernando, Del Indianismo a la Indianidad. Desarrollo histórico de la teología india (1989), 56.

46 Moore Laurence Beem Beverly e Benton Roy, et al. pectrum Reaches Of International Relations (1990), 50. 
sin discriminación, explotación, humillación y con derecho a educación, el educando debe ser consciente de su realidad y cambiar su estructura mental para su transformación y liberación mediante un pensamiento crítico de su realidad circundante. En esencia, la educación liberadora centra su atención en la concientización e interpretación, la comprensión crítica y la acción transformadora de su comunidad.

Manuel Zúñiga Camacho fue el primer impulsor de la educación rural en el Perú y precursor de la educación liberadora en América Latina. Fue la primera escuela en el Perú y en América para niños indios y con profesor indígena. Se empezó la labor de alfabetización, para liberar al campesinado, enseñando a la gente a preservarse de los males mediante la higiene personal, en la escuela de Utawilaya empezó la liberación de las conciencias. Después de 58 años de ardua lucha surge en 1961 en Latinoamérica la teoría pedagógica de Paulo Freire sustentada en su obra "Pedagogía de la Liberación" pedagogía del oprimido (1970) "Educación como práctica de la libertad" y otras interesadas en la problemática educativa.

\section{REFERENCIAS}

Aliaga Rodríguez, Gustavo. El Rijcharismo en el Altiplano peruano como una experiencia pionera de salud intercultural en América. "Rijcharismo" movement in the Peruvian High Plateau as a Pioneer Intercultural Health Experience in the Americas., 2015. 497509. Retrieved from http://search.ebscohost.com/login.aspx?direct=true\&db=a9h \&AN=109373962\&lang=es\&site=ehost-live.

Allpanchis. Poder y Autoridad en los Andes. Sicuani - Cusco: Instituto de Pastoral Andina, (1989).

Alomia, Merling. Un Importante Centenario en la Historia de las Misiones con su Espíritu, 1998. 61.

Basadre Grohmann, Jorge. Historia de la República del Perú (1822-1933) (Quinta ed., Vol. 10). Lima, Perú, Ediciones Historia, 1963.

Burga, Manuel \& Flores Galindo Alberto. Apogeo y crisis de la República Aristocrática. Lima, Ediciones Rikchay Perú, 1990.

Cano, LA UNIÓN. Lo de la Platería, julio (23 de enero de 1914), 2.

CARE. Fichas botánicas de especies agroforestales. Paz: CARE y Programa Nacional de Cambio, 2011.

Cabrera, Isaac Irán \& Gallardo, Teresita. Educación intercultural del estudiante universitario: el enfoque de formación humanístico intercultural. Revista Electrónica "Actualidades Investigativas en Educación" - Universidad de Costa Rica, (2013). pp. 1-34. 
Ccahuana Córdova, Jorge Alberto. Repositorio de tesis de la Pontificia Universidad Católica del Perú. (5 de noviembre de 2013). http://tesis.pucp.edu.pe/repositorio/bitstream/ handle/123456789/5126/CCAHUANA_CORDOVA_JORGE_CAPACIDAD_ INDIGENA.pdf?sequence=1

Citarela Luca, Amadio Massimo, \& Zúñiga, Madeleine. La educación indígena en América Latina, vol. Tres, Quito, Ecuador, ABYA AYALA - UNESCO, 1990.

Colin, Lankshear and Knobel, Michele. Tres Enfoques para la Investigación Educativa. México: UNAM. (2002).

Comboni Salinas, Sonia \& Juárez Núñez, José Manuel. Diversidad cultural, educación y democracia: etapas en la construcción de la educación indígena en América Latina. Educação \& Sociedade, ano XXII, no 7, 2001. 235 - 274.

Ferrão Candau, Vera María. (2010). Educación intercultural en América Latina: distintas concepciones y tensiones actuales. SCIELO Chile. Estudios Pedagógicos XXXVI, No. 2, 333-342.

Gallegos Arriola, Luis. El Campesino Rebelde del Altipampa. Puno, Perú: Centro de estudios y Reflexiones, 1974.

Gallegos Arriola, Luis. Biografía de un Aymara. Puno: Universitaria, 1993.

INEI. "Informe técnica: evolución de la pobreza al 2009". Lima: INEI, 2010.

INEI. (01 de mayo de 2014). INEI. 03 de mayo de 2016, https://www.inei.gob.pe/media/ cifras_de_pobreza/informetecnico.pdf

Jove Quimper, Hernán Alberto. Poder Patrimonial y dominio Iglesia “Tintiri”, Azangaro Puno: 1860-1968. Lizares terrateniente de Azangaro: Fanático religioso, maniático por el dinero y despojador de tierras. Rev. Investig. Altoandin. 2016; Vol. 18, No. 1: 93 - 102, Vol 18, No. 1: 93 - 102.

Kapsoli Escudero, Wilfredo. Los Movimientos Campesinos en el Perú 1879-1965. Lima, Perú, Delva Editores, 1977.

Kessler, J. A. Historia de la Evangelización en el Perú. Lima: Puma, 1993.

Málaga Sabogal, Ximena. (14 de octubre de 2014). Pontificia Universidad Católica del Perú. Obtenido de: http://tesis.pucp.edu.pe/repositorio/bitstream/ handle/123456789/5716/MALAGA_SABOGAL_XIMENA_EDUCACION_ PUNO.pdf?sequence=1

Mariátegui La Chira, José Carlos. 7 ensayos de interpretación de la realidad peruana. (Vol. I). Ayacucho, Perú: Fundación Biblioteca Ayacucho, 1979.

Mires, Fernando. Del Indianismo a la Indianidad. Desarrollo histórico de la teología india, 1989.

MPP. (20014). Plan Estrategico Institucional. Obtenido de: www.munipuno.gob.pe/ descargas/.../plan_estrategico_institucional.pdf

Núñez Núñez, Héctor Elías. (2008). UNMSM. Obtenido de Tesis: http://cybertesis. unmsm.edu.pe/bitstream/cybertesis/3113/1/Nu\%C3\%B1ez_nh.pdf 
Portugal Catacora, José. (30 de octubre de 2012). Blog Portugal. Recuperado el 01 de mayo de 2016, de: http://joseportugalcatacora.blogspot.pe/2012/10/manuel-zcamacho.html

Portugal Catacora, José. Historia de la Educación en Puno. Puno, Perú: Corporación MERU E.I.R.L., 2013.

Rengifo, Grimaldo Antonio. Campesinos en Puno 1895 - 1925 Grimaldo Antonio Rengifo Balarezo. Tesis para optar el Título de Lima - 1990.

Rénique, José Luis \& Deustua, José. Intelectuales, indigenismo y descentralismo en el Perú, 1897-1931. Cusco, Perú: Centro Bartolomé de las Casas, 1984.

Sandoval Zúñiga, Oscar Raúl. El problema de la tierra y la cuestión indígena en la obra siete ensayos de interpretación de la realidad peruana de José Carlos Mariátegui. PORIK AN, 271 - 28, 2004.

Spalding, Karen. De indios a campesinos. Lima: Instituto de Estudios Peruanos, 1974.

Tamayo Herrera, José. Historia social e indigenismo en el altiplano. Lima, Perú: Trentaitrés, 1982.

Tamayo Tamayo, Mario. Aprender a investigar. Cali - Colombia: ICFES, 1998.

UNICEF. Estado de la Niñez en el Perú. Lima, Perú: Asociación Gráfica Educativa, 2011.

Rengifo, Grimaldo Antonio. Campesinos en Puno 1895 - 1925 Grimaldo Antonio Rengifo Balarezo. Tesis para optar el Título de Lima - 1990.

Ruelas Vargas, David. "La escuela rural de Utawilaya: Una Educación liberadora desde Puno - Perú 1902". Revista Historia de la Educación Latinoamericana. Vol. 18 No. 27 (2016): 243-262. 